\title{
Normative ranges of anthropometric cranial indices and metopic suture closure during infancy
}

\author{
Jonathan Pindrik, MD, ${ }^{1}$ Joseph Molenda, MD, ${ }^{1}$ Rafael Uribe-Cardenas, MD, MHS, ${ }^{1}$ \\ Amir H. Dorafshar, MBChB, ${ }^{2}$ and Edward S. Ahn, MD' \\ 1Division of Pediatric Neurosurgery, Department of Neurosurgery, and 2Department of Plastic and Reconstructive Surgery, Johns \\ Hopkins University School of Medicine, Baltimore, Maryland
}

\begin{abstract}
OBJECTIVE Subjective evaluations typically guide craniosynostosis repair. This study provides normative values of anthropometric cranial indices that are clinically useful for the evaluation of multiple types of craniosynostosis and introduces 2 new indices that are useful in the evaluation and management of metopic and bicoronal synostosis. The authors hypothesize that normative values of the new indices as well as for established measures like the cephalic index can be drawn from the evaluation of CT scans of normal individuals.
\end{abstract}

METHODS High-resolution 3D CT scans obtained in normal infants (age 0-24 months) were retrospectively reviewed. Calvarial measurements obtained from advanced imaging visualization software were used to compute cranial indices. Additionally, metopic sutures were evaluated for patency or closure.

RESULTS A total of 312 participants were included in the study. Each monthly age group (total 24) included 12-18 patients, yielding 324 head CT scans studied. The mean cephalic index decreased from 0.85 at age $0-3$ months to 0.81 at 19-24 months, the mean frontoparietal index decreased from 0.68 to 0.65 , the metopic index from 0.59 to 0.55 , and the towering index remained comparatively uniform at 0.64 and 0.65 . Trends were statistically significant for all measured indices. There were no significant differences found in mean cranial indices between sexes in any age group. Metopic suture closure frequency for ages 3, 6, and 9 months were $38.5 \%, 69.2 \%$, and $100.0 \%$, respectively.

CONCLUSIONS Radiographically acquired normative values for anthropometric cranial indices during infancy can be used as standards for guiding preoperative decision making, surgical correction, and postoperative helmeting in various forms of craniosynostosis. Metopic and towering indices represent new cranial indices that are potentially useful for the clinical evaluation of metopic and bicoronal synostoses, respectively. The present study additionally shows that metopic suture closure appears ubiquitous after 9 months of age.

http://thejns.org/doi/abs/10.3171/2016.5.PEDS14336

KEY WORDS craniosynostosis; craniometric indices; cephalic index; metopic synostosis; metopic index; towering index; craniofacial

$\mathrm{C}$ RANIOSYNOSTOSIS represents premature fusion of one or multiple cranial sutures. This condition may lead to noticeable craniofacial deformity and infrequently may impact neurological development or intracranial pressure. Surgical repair of craniosynostosis usually requires a collaborative approach between pediatric neurosurgery and plastic surgery or craniofacial reconstructive teams. Aesthetic markers and subjective assessments typically guide clinical evaluation, operative planning, reconstruction, and postoperative assessment or management. While clinically useful, these current methods lack objective standards to evaluate patients with craniosynostosis pre- and postoperatively.

Anthropometric cranial measurements provide objective and reproducible techniques for evaluating the human calvaria. Normal reference ranges of cranial measurements

ABBREVIATIONS EUD = eurion-eurion diameter; $\mathrm{GOPD}$ = glabella-opisthocranion diameter; $\mathrm{GOPP}$ = glabella-opisthocranion perimeter; $\mathrm{MFZD}=$ midfrontozygomatic diameter.

SUBMITTED August 3, 2014. ACCEPTED May 25, 2016.

INCLUDE WHEN CITING Published online September 2, 2016; DOI: 10.3171/2016.5.PEDS14336. 
TABLE 1. Calvarial anatomical landmarks

\begin{tabular}{|c|c|}
\hline $\begin{array}{c}\text { Anatomical } \\
\text { Landmark }\end{array}$ & Description \\
\hline Glabella* & $\begin{array}{l}\text { Anterior extreme in midsagittal plane at lower mar- } \\
\text { gin of the frontal bone, above frontonasal suture, } \\
\text { \& between superciliary arches }\end{array}$ \\
\hline Opisthocranion* & $\begin{array}{l}\text { Posterior extreme in midsagittal plane on superior } \\
\text { squamous of occipital bone; may or may not } \\
\text { coincide with external occipital protuberance }\end{array}$ \\
\hline Eurion* & $\begin{array}{l}\text { Lateral extreme of the skull on either parietal bone } \\
\text { or upper temporal bone (avoiding any lower } \\
\text { temporal protrusion or bulge) }\end{array}$ \\
\hline Frontotemporal* & $\begin{array}{l}\text { Most medial indentation of the inferolateral frontal } \\
\text { bone, superolateral to the brow ridge, immedi- } \\
\text { ately posterior to the zygomatic process of the } \\
\text { frontal bone, \& anterior to the pterion }\end{array}$ \\
\hline MFZ recess $†$ & $\begin{array}{l}\text { Midpoint along the recess above the brow ridge } \\
\text { between the anteroinferolateral frontal bone \& } \\
\text { frontal bone zygomatic process }\end{array}$ \\
\hline
\end{tabular}

* Conventional landmark.

$\dagger$ Newly defined anatomical landmark.

can offer quantitative standards for diagnosing craniosynostosis and craniofacial deformities objectively and reliably.,16,17 This study explores radiographically acquired normative values of cranial indices, based on anthropometric measurements, in healthy full-term children 0 to 24 months of age. We additionally explore the patency and closure frequencies of the metopic suture within each age group studied. We hypothesize that normative ranges of cranial indices, including 2 novel cranial indices proposed in this work, can be extracted from the analysis of 3D CT scans and used as references applicable to multiple types of craniosynostosis.

\section{Methods}

After obtaining institutional review board approval, high-resolution, reconstructed 3D head CT scans obtained in 0- to 24-month-old normal full-term infants were retrospectively reviewed by 2 observers (J.P. and J.M.). Radiographic images had been acquired previously at our institution for clinical purposes to evaluate trauma, concern for nonaccidental trauma, headache, seizures, changes in neurological status or level of consciousness, and/or abnormalities in neurological examination findings. Serial imaging in the same study subject would not be incorporated unless consecutive images were obtained at least 1 month apart. The following conditions represented exclusion criteria for study subjects: prematurity, hydrocephalus (treated or untreated), intracranial tumors or mass lesions, intracranial arachnoid cysts, intracranial hemorrhage, craniosynostosis, skeletal disease affecting the calvaria (e.g., osteogenesis imperfecta), any growth-related syndromes (e.g., congenital hyper- or hypothyroidism), congenital ischemic encephalopathy, skull fractures affecting midline calvarial measurements, or any other condition potentially affecting cranial size or shape, including positional plagiocephaly.
TABLE 2. Anthropometric cranial measurements

\begin{tabular}{lll}
\hline \multicolumn{1}{c}{ Cranial Measurement } & Term & \multicolumn{1}{c}{ Description } \\
\hline $\begin{array}{l}\text { Glabella-opisthocranion } \\
\text { diameter }\end{array}$ & GOPD & Maximum cranial AP length \\
\hline $\begin{array}{l}\text { Glabella-opisthocranion } \\
\text { perimeter }\end{array}$ & GOPP & Maximum cranial AP perimeter \\
\hline Eurion-eurion diameter & EuD & Maximum cranial width (breadth) \\
\hline Frontotemporal diameter & FTD & Minimal frontotemporal width \\
\hline $\begin{array}{l}\text { Midfrontozygomatic } \\
\text { diameter }\end{array}$ & MFZD & Minimal frontozygomatic width \\
\hline AP = anteroposterior. & & \\
\hline
\end{tabular}

$\mathrm{AP}=$ anteroposterior.

Specific head CT parameters included slice thickness at or below $1 \mathrm{~mm}$ and the ability to evaluate high-resolution 3D reconstructions (using bone windows). Observers used UltraVisual Advanced Visualization software (Emageon) to reconstruct and evaluate 3D images. Frontal projections allowed determination of metopic suture patency or closure. Superior (bird's-eye view over the top of the calvaria) and lateral projections allowed linear measurement between predefined anatomical landmarks (Table 1) using the geometrical "polygon tool." Lateral projections of the calvaria with clear visualization of anatomical landmarks were exported to Synedra View Personal 3 (Synedra Information Technologies $\mathrm{GmbH}$ ) to measure curvilinear distances with the "measure polygon" function. Measurements acquired from lateral projections were performed along the midsagittal plane. We designated specific anthropometric cranial measurements based on standard conventions or newly defined dimensions (Table 2). ${ }^{2,5,6}$ Based on linear and curvilinear distances acquired, specific cranial indices were computed (Table 3).

Study subjects were stratified by age without separation by sex. Cranial indices of study subjects were compiled and analyzed using descriptive statistics across age groups. Subsequently, sex stratification of the study population allowed comparative analysis between sexes using the unpaired Student t-test. Nonparametric testing was performed to evaluate the significance of trends of the different cranial indices across age categories. To assess reliability, intraclass correlation coefficients were calculated on a random $20 \%$ subsample of the total population using a 2-way mixed-effects model. Congruent with prior studies, we defined metopic suture closure as fusion or os-

\section{TABLE 3. Anthropometric cranial indices}

\begin{tabular}{|c|c|c|}
\hline Cranial Index & Ratio & Proposed Applicability \\
\hline Cephalic index* & $\begin{array}{l}\text { EuD/ } \\
\text { GOPD }\end{array}$ & $\begin{array}{l}\text { Sagittal synostosis, dolichocepha- } \\
\text { ly, brachycephaly }\end{array}$ \\
\hline Frontoparietal index* & FTD/EuD & Metopic synostosis, trigonocephaly \\
\hline Metopic index† & MFZD/EuD & Metopic synostosis, trigonocephaly \\
\hline Towering index $\dagger$ & $\begin{array}{l}\text { GOPD/ } \\
\text { GOPP }\end{array}$ & $\begin{array}{l}\text { Bilateral coronal synostosis, tur- } \\
\text { ricephaly }\end{array}$ \\
\hline
\end{tabular}

FTD = frontotemporal diameter.

* Based on standard conventions.

$\uparrow$ Newly defined anthropometric cranial index. 


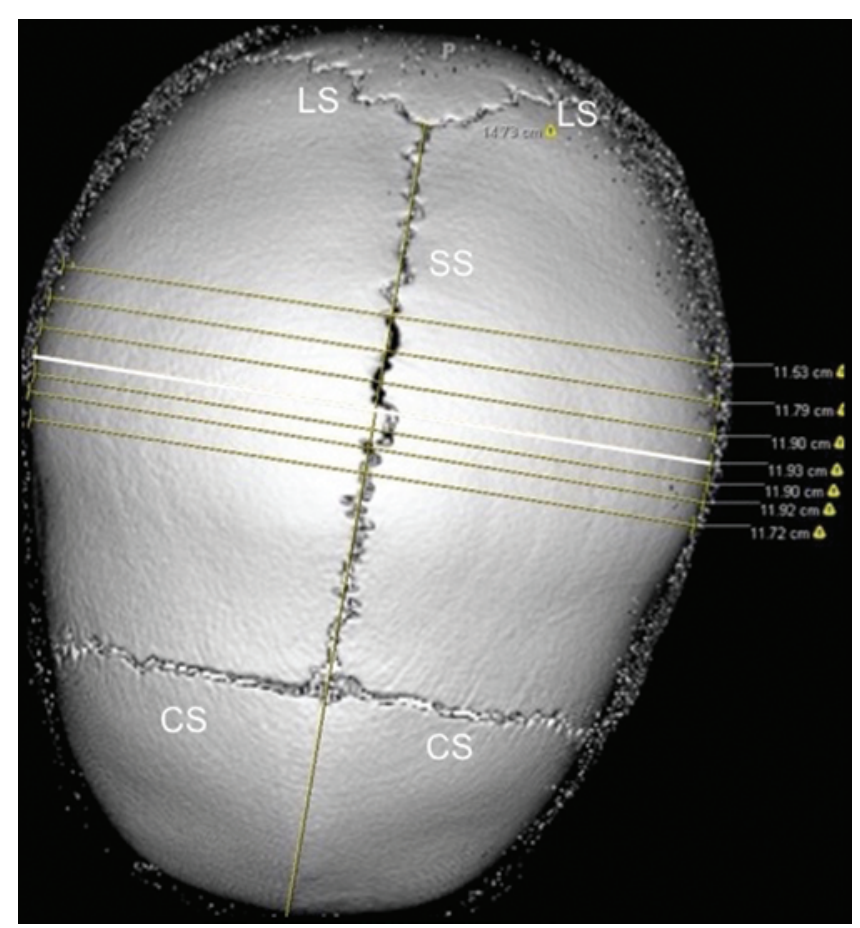

FIG. 1. Anthropometric cranial measurement, EuD. This superior projection of a 3D head CT reconstruction displays the measurement of the EuD. Several trial measurements were performed to define the greatest biparietal diameter (119.3 $\mathrm{mm}$ in this case). CS = coronal suture; LS = lambdoid suture; SS = sagittal suture. Figure is available in color online only.

sification of the metopic suture line without radiographic evidence of suture patency between the frontonasal suture and ventral extreme of the anterior fontanelle..$^{14}$ The frequency of metopic suture closure was calculated for each monthly age group.

\section{Results}

Between 12 and 18 subjects were evaluated within each monthly age group (0-24 months), totaling 324 head CT scans obtained in 312 patients. The study population consisted of a near-equal distribution across sexes (male $52.2 \%$ and female $47.8 \%$ ). The most common indications for imaging included trauma, seizures, concern for nonaccidental trauma, altered mental status or loss of consciousness, headaches or emesis, and concern for skull or scalp abnormalities based on physical examination findings.

Rotation of 3D reconstructed images allowed acquisition of predefined anthropometric cranial measurements (Figs. 1-3). Stratification of the study population into age groups allowed compilation and comparison of mean anthropometric cranial indices (Table 4). The mean cephalic index decreased from 0.85 (SD 0.05) at age 0-3 months to $0.81(0.05)$ at age $19-24$ months. The mean frontoparietal index decreased from $0.68(0.03)$ at age $0-3$ months to $0.65(0.03)$ at age 7-24 months. The mean metopic index (Fig. 2) decreased from $0.59(0.02)$ at age $0-3$ months to $0.55(0.03)$ at age 13-24 months. The mean towering index
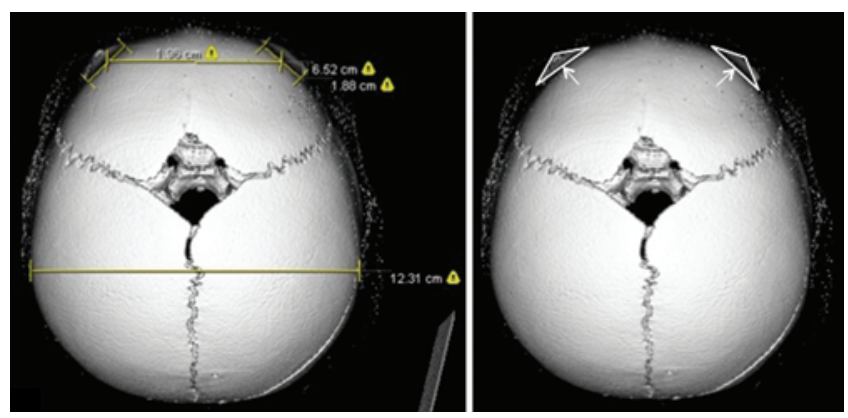

FIG. 2. MFZD and metopic index. Left: This superior projection of a 3D head CT reconstruction displays the measurement of MFZD $(65.2 \mathrm{~mm}$ in this example). Division of this value (MFZD) by the maximum cranial width, or EuD (123.1 mm), yields the metopic index (in this example, 0.53). Right: The triangular disposition of the orbital rims (triangles) used to calculate the MFZD. The arrows point to the midfrontozygomatic recess. The MFZD is the resulting diameter that connects those points. Figure is available in color online only.

(Fig. 3) remained comparatively uniform from 0.64 (0.02) at age $0-12$ months to $0.65(0.02)$ at age 13-24 months. Sex stratification revealed similar mean anthropometric cranial indices for both sexes across all ages without significant differences (Table 5); a statistically significant trend was found for each of the cranial indices across all age groups (Table 4). Intraclass correlation coefficients were used to assess reliability of measurements using a 2-way mixedeffects model and estimated at 0.8 (95\% CI 0.62-0.89, $\mathrm{p}<$ $0.001)$ for the metopic index, $0.4(95 \%$ CI $0.04-0.64, \mathrm{p}=$ 0.014 ) for the towering index, and 0.8 (95\% CI 0.64-0.90, $\mathrm{p}<0.001)$ for metopic suture closure.

No imaging studies obtained in 0 - to 2-month-old normal infants reflected fusion of the metopic suture. Metopic suture closure frequency reached $38.5 \%$ at 3 months, $69.2 \%$ at 6 months, and $100.0 \%$ at and beyond 9 months (Table 6).

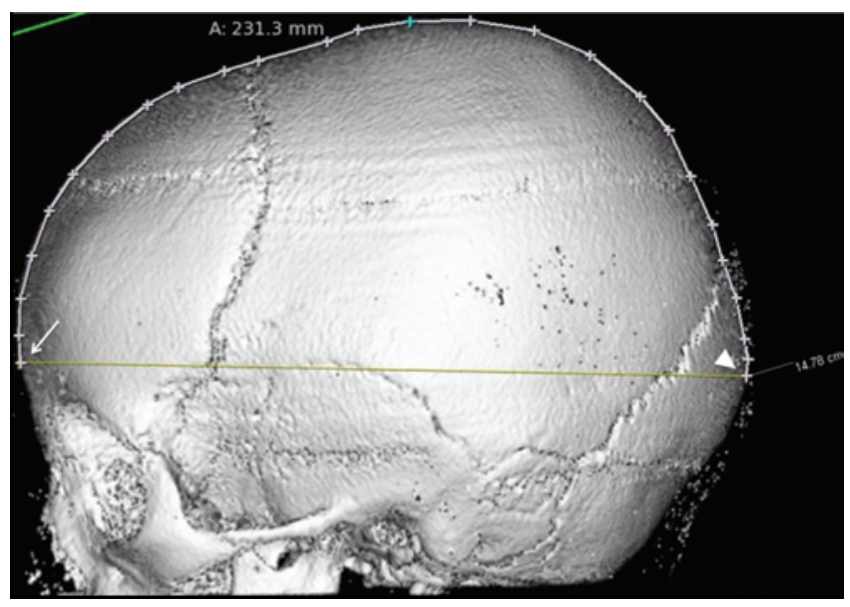

FIG. 3. Towering index. This lateral projection of a 3D head CT reconstruction displays the measurement of the GOPD and GOPP. The arrow indicates the glabella and the arrowhead the opisthocranion. In this example, GOPD $=147.8 \mathrm{~mm}$ and GOPP $=231.3 \mathrm{~mm}$, yielding a towering index (GOPD/GOPP) of 0.64 . Figure is available in color online only. 
TABLE 4. Mean anthropometric cranial indices by age group*

\begin{tabular}{lcccc}
\hline Age Group (mos) & Cephalic Index & Frontoparietal Index & Metopic Index & Towering Index \\
\hline $0-3$ & $0.85(0.75-0.95)$ & $0.68(0.62-0.74)$ & $0.59(0.55-0.63)$ & $0.64(0.60-0.68)$ \\
\hline $4-6$ & $0.84(0.74-0.94)$ & $0.66(0.60-0.72)$ & $0.58(0.54-0.62)$ & $0.64(0.58-0.70)$ \\
\hline $7-12$ & $0.83(0.73-0.93)$ & $0.65(0.59-0.71)$ & $0.56(0.50-0.62)$ & $0.64(0.60-0.68)$ \\
\hline $13-18$ & $0.82(0.72-0.92)$ & $0.65(0.59-0.71)$ & $0.55(0.49-0.61)$ & $0.65(0.61-0.69)$ \\
\hline $19-24$ & $0.81(0.71-0.91)$ & $0.65(0.59-0.71)$ & $0.55(0.49-0.61)$ & $0.65(0.61-0.69)$ \\
\hline Trend p value) & $<0.001$ & $<0.001$ & $<0.001$ & 0.011 \\
\hline
\end{tabular}

* All non- $p$ values are reported as the mean (range), where range $=$ mean $\pm 2 *[S D]$.

\section{Discussion}

Craniosynostosis indicates premature fusion of one or multiple cranial sutures (sagittal, coronal, metopic, or lambdoid). Premature suture fusion may reflect an isolated abnormality or underlying dysmorphogenic syndrome such as Crouzon's or Alpert's syndrome. Coronal and metopic synostoses commonly exhibit multiple associated craniofacial abnormalities. ${ }^{4,9}$ In addition to cosmetic deformity, craniosynostosis may rarely cause elevated intracranial pressure or contribute to behavioral abnormalities and developmental delay. ${ }^{14}$ In the context of these abnormalities, affected patients may undergo operative craniosynostosis repair and craniofacial reconstruction.

The surgical techniques employed for craniofacial reconstruction have evolved over time, while the methods of assessment have remained fairly constant. ${ }^{11}$ Subjective assessments by the craniofacial reconstructive or pediatric neurosurgical teams typically guide preoperative consideration and surgical planning. Similarly, postoperative evaluation and management of patients undergoing craniofacial reconstruction often employ subjective criteria. ${ }^{3,8}$ While useful, these aesthetic markers for operative decision making and postoperative management lack objectivity and quantified standards. The most commonly used Whitaker classification system assesses whether the patient, family, or surgeon considers operative revision advisable or necessary. ${ }^{15}$ Objective standards based on craniometric measurements provide more reliable measures of outcome and could guide the pre- and postoperative management of craniosynostosis. Other types of craniofacial deformity, including positional plagiocephaly and brachycephaly, could also benefit from objective parameters of evaluation. ${ }^{12}$

Definition of a large series of developmentally normal, full-term infants with high-resolution 3D reconstructed head CT scans allowed exploration of normative ranges for common anthropometric cranial indices and the development of 2 new indices (metopic index and towering index) that are potentially useful in the clinical setting. The same series of imaging was used to assess timing of metopic suture closure among infants. These normative values provide references that are potentially applicable to multiple types of craniosynostosis, including sagittal, bicoronal, and metopic synostoses. Normative values of anthropometric cranial indices offer objective standards to help guide craniosynostosis repair with respect to preoperative decision making, surgical planning, and postoperative helmeting.

\section{Anthropometric Cranial Indices}

Multiple authors have reported manually obtained anthropometric cranial measurements or indices in children and adults. Christofides and Steinmann described their production of a head shape chart based on the glabellaopisthocranion diameter (GOPD), eurion-to-eurion diameter (EuD; i.e., the maximum cranial width), and ear-to-ear length in infants and adults. In addition to plotting cranial growth trends for individual patients postoperatively, the chart could be used to qualify abnormal head shapes (such as scaphocephaly and brachycephaly) based on quantitative data of cephalic index and ear-to-ear measurements. ${ }^{2}$ Of note, the cephalic indices based on work by Farkas et al. and reported by Christofides and Steinmann appear slightly lower $(0.73-0.79)$ than those in the present study (0.81-0.83) within ages 6-24 months., ${ }^{2,6}$ It is possible that these differences could be accounted for by differences in measurement technique since both of those reports used surface measurements to calculate maximal cranial length and width. In younger children it is possible that measurements might not be as accurate since it is difficult for them to remain still during evaluation. In addition to this, using surface measurements is theoretically more prone to error based on the fact that certain landmarks might not be equally evident in all patients (e.g., the external occipital protuberance or the cranial vertex) or that shifting of the skin might falsely increase or decrease a specific measure. In a similar study, Wilbrand et al. evaluated the cephalic index in normal infants (age 0-12 months) and children with nonsynostotic cranial deformity to help determine objective standards for diagnosing positional plagiocephaly, brachycephaly, or both. Based on manually acquired anthropometric cranial measurements, these authors reported 50th percentile cephalic indices (0.79-0.84) in normal children, which closely approximates the results of our study (Table 4). ${ }^{16}$ Other authors have reported similar normative cephalic index ranges of $0.75-0.85$ in prior articles. ${ }^{10}$ In contrast to multiple studies investigating cephalic index, few articles have reported quantitative data regarding the normal facio-orbital complex or aberrations seen in metopic synostosis. ${ }^{9}$

Deviation of anthropometric cranial indices from normative values may adequately reflect structural abnormalities of the calvaria. Multiple studies have justified the application of anthropometric cranial measurements and proportions in craniofacial deformity. Asha et al. reported mean elevated cephalic index $(0.88)$ in southern Indian children with Down syndrome, quantifying the craniofacial dysmorphogenesis iconic to this group of pa- 
TABLE 5. Mean anthropometric cranial indices for all ages (0-24 months) by sex*

\begin{tabular}{lllll}
\hline \multicolumn{1}{c}{ Sex } & $\begin{array}{c}\text { Cephalic } \\
\text { Index }\end{array}$ & $\begin{array}{c}\text { Frontoparietal } \\
\text { Index }\end{array}$ & $\begin{array}{c}\text { Metopic } \\
\text { Index }\end{array}$ & $\begin{array}{c}\text { Towering } \\
\text { Index }\end{array}$ \\
\hline Male & $0.82(0.05)$ & $0.66(0.03)$ & $0.57(0.04)$ & $0.64(0.02)$ \\
\hline Female & $0.83(0.05)$ & $0.65(0.03)$ & $0.57(0.04)$ & $0.65(0.02)$ \\
\hline p value† & 0.52 & 0.12 & 0.71 & 0.15 \\
\hline
\end{tabular}

* All means are reported with SD.

$†$ Based on an unpaired Student t-test.

tients. ${ }^{1}$ Comparing children with positional brachycephaly to normal infants, Wilbrand et al. demonstrated that more than $99 \%$ of affected patients exhibited cephalic indices greater than the 90th percentile. ${ }^{16}$ In a study evaluating children with isolated craniosynostosis, Farkas et al. showed that coronal synostosis exhibited a stronger impact on anthropometric cranial measurements rather than facial or orbital dimensions. ${ }^{5}$ In a similar study that investigated children with nonsyndromic (isolated) metopic synostosis, Kolar and Salter demonstrated abnormal reduction of the frontoparietal index in affected patients as compared with normal standards. While the cephalic index of these subjects reflected normal values, the lower frontoparietal index quantified the aberrant cranial geometry typical for trigonocephaly in metopic synostosis. ${ }^{9}$ The profound impact of craniofacial dysmorphogenesis on structural aberrations of the calvaria justifies the use of anthropometric cranial measurements to characterize patients with craniofacial deformity and craniosynostosis.

Anthropometric cranial measurements offer objective, reproducible methods of evaluating craniofacial deformity and craniosynostosis with adequate clinical validity. ${ }^{16,17}$ Several studies have demonstrated low intra- and interobserver variability of these simple and repeatable measurements when implementing standardized protocols. ${ }^{17}$ Many authors agree that objective cranial measurements represent an integral component in the evaluation and treatment of craniofacial deformity. ${ }^{1,2,5,13,17}$ Analysis of cranial measurements from individual patients requires comparison with a range of normative values. Despite reporting manually acquired anthropometric cranial measurements in large groups, few studies have described radiographic norms of head shape and/or size. ${ }^{2,5}$ This study provides normative ranges of anthropometric cranial indices acquired from high-resolution head CT scans obtained in healthy full-term children (Table 4). Based on measurements obtainable both radiographically and clinically, these cranial indices offer potential applications in the evaluation and management of different types of craniosynostosis (Table 3 ).

\section{New Clinically Useful Cranial Indices: Metopic Index and Towering Index}

In addition to standard cranial indices described previously (cephalic index and frontoparietal index), we propose 2 novel cranial indices, metopic index and towering index, applicable to the evaluation of metopic and bilat-
TABLE 6. Metopic suture closure frequency by age

\begin{tabular}{ccc}
\hline Age (mos) & $\begin{array}{c}\text { Sample Size } \\
\text { (no. of patients) }\end{array}$ & $\begin{array}{c}\text { Metopic Suture } \\
\text { Closure Frequency }\end{array}$ \\
\hline $0-2$ & 45 & $0.0 \%$ \\
\hline 3 & 13 & $38.5 \%$ \\
\hline 4 & 13 & $46.2 \%$ \\
\hline 5 & 18 & $61.1 \%$ \\
\hline 6 & 13 & $69.2 \%$ \\
\hline 9 & 12 & $100.0 \%$ \\
\hline 10 & 183 & $100.0 \%$ \\
\hline
\end{tabular}

eral coronal synostoses, respectively. Of note, the normative means for metopic index $(0.55-0.59)$ fall below the normative means for frontoparietal index $(0.65-0.68)$, reflecting measurement along a more anteriorly located, narrower portion of the skull that may better describe the aberrant fronto-orbito-zygomatic complex in trigonocephaly. ${ }^{9}$ Providing the foundation for measurement of frontoparietal index, the frontotemporal ridge does not exhibit as severe displacement in cases of metopic craniosynostosis. Additionally, clinicians often encounter difficulty when attempting to palpate the frontotemporal ridge. We propose that measurement along the brow ridge at the midfrontozygomatic recess more adequately reflects the narrowing present in metopic craniosynostosis. This point also is visualized easily in the clinical setting by viewing the head from above and locating the brow ridge (Fig. 2), accentuated by the narrowed forehead in trigonocephaly.

Given the physiological fusion of the metopic suture during the 1st year of life, as demonstrated in our study, concerns for metopic craniosynostosis commonly prompt evaluation by craniofacial surgeons. In such cases, clinicians must determine if a child demonstrates frontal narrowing that lies outside the norm. By reporting mean values and ranges (determined by 2 standard deviations above and below the mean) for each cranial index, we propose that ratios falling outside the range may indicate abnormal cranial geometry. Therefore, the metopic index may provide utility in determining candidates who may benefit from surgical correction of trigonocephaly. Similarly, our reported values of cephalic index with ranges determined by standard deviation may guide treatment of children with mild to moderate scaphocephaly due to only partial fusion of the sagittal suture. Furthermore, the reported mean cranial indices can be used as potential standards to guide operative planning, intraoperative reconstruction, and postoperative helmeting in multiple types of craniosynostosis.

The towering index, a cranial index, reflects the abnormal towering and vertical elongation of the calvaria by comparing the net distance to arc length between the glabella and opisthocranion. For this study based on 3D head CT reconstructions, the glabella and opisthocranion were determined uniformly among observers. However, in the clinical setting, we recognize that variability may exist in the glabella-opisthocranion dimensions between measurers. Therefore, an alternative measure of towering 
may be performed more reliably in the clinic setting. Measurement of the maximum perimeter between each tragus of the ear, over top of the calvarial vault, may provide less variance due to better recognition of external landmarks. Therefore, towering index in the clinical setting may be defined more reliably as the maximum tragus to tragus perimeter divided by the occipitofrontal circumference (OFC), both routinely obtainable on physical examination. However, since this study used 3D reconstructions of bone windows only, measurements were not performed based on external soft-tissue landmarks. A future study comparing these methods of measurement, particularly in the setting of bilateral coronal craniosynostosis, may provide utility for more direct clinical application.

\section{Metopic Suture Closure}

The frequency of metopic suture closure reflected a generally smooth increase with respect to age (Table 6). Metopic suture closure frequency first reached above $0 \%$ at age 3 months (38.5\%) and surpassed 50\% at age 5 months (61.1\%). All study subjects 9 months of age or older exhibited a closed metopic suture. These findings closely reflect results of prior studies reporting metopic suture closure frequencies of $33 \%$ at age 3 months, $59 \%$ at 5 months, and $100 \%$ at or beyond 9 months. ${ }^{14}$ Recognition of these reference values describing normal timing of metopic suture closure may help selectively define aberrations. Although accounting for a minority (3\%-5\%) of craniosynostoses, premature metopic suture fusion leading to trigonocephaly typically requires aggressive surgical correction. ${ }^{9,14}$

\section{Study Limitations}

Although many high-resolution 3D reconstructed head CT scans were evaluated for this study, the incorporation of more subjects within each age group would improve generalizability of the study sample to the pediatric population. Furthermore, review of the head CT scans by more than 2 observers, with a method to average or correct for any appreciable interobserver error, could improve data analysis. The extension of this mode of radiographic analysis to patients with craniosynostosis and high-resolution imaging represents a fitting next step of inquiry. This would allow comparison of cranial indices in patients with craniofacial deformity to those with normal craniofacial structure and investigate any noteworthy differences from the cranial indices reported here. Nevertheless, the presentation of normative ranges of cranial indices in pediatric patients $0-24$ months of age represents a worthwhile first step in providing objective standards for craniosynostosis evaluation and repair.

\section{Conclusions}

Subjective assessments and aesthetic markers typically guide evaluation of and surgical planning for multiple types of craniosynostosis. Few objective standards currently exist to define structural aberrations from the norm and help plan appropriate therapy. This study presents a simple and reliable method, applicable both radiographically and clinically, of measuring common anthropometric cranial indi- ces useful in the evaluation of craniofacial deformities. We also introduce 2 new cranial indices (metopic index and towering index) that are potentially applicable to metopic and bicoronal synostoses. Radiographically acquired normative values for anthropometric cranial indices during infancy can be used as standards for guiding preoperative decision making, surgical correction, and postoperative helmeting in various forms of craniosynostosis.

\section{References}

1. Asha KR, Lakshmiprabha S, Nanjaiah CM, Prashanth SN: Craniofacial anthropometric analysis in Down syndrome. Indian J Pediatr 78:1091-1095, 2011

2. Christofides EA, Steinmann ME: A novel anthropometric chart for craniofacial surgery. J Craniofac Surg 21:352-357, 2010

3. Collett BR, Gray KE, Kapp-Simon KA, Birgfeld C, Cunningham M, Rudo-Stern J, et al: Laypersons' ratings of appearance in children with and without single-suture craniosynostosis. J Craniofac Surg 24:1331-1335, 2013

4. Delashaw JB, Persing JA, Broaddus WC, Jane JA: Cranial vault growth in craniosynostosis. J Neurosurg 70:159-165, 1989

5. Farkas LG, Katic MJ, Forrest CR: Anthropometric proportion indices in the craniofacial regions of 73 patients with forms of isolated coronal synostosis. Ann Plast Surg 55:495-499, 2005

6. Farkas LG, Posnick JC, Hreczko TM: Anthropometric growth study of the head. Cleft Palate Craniofac J 29:303308, 1992

7. Hankinson TC, Fontana EJ, Anderson RC, Feldstein NA: Surgical treatment of single-suture craniosynostosis: an argument for quantitative methods to evaluate cosmetic outcomes. J Neurosurg Pediatr 6:193-197, 2010

8. Hilling DE, Mathijssen IM, Vaandrager JM: Aesthetic results of fronto-orbital correction in trigonocephaly. J Craniofac Surg 17:1167-1174, 2006

9. Kolar JC, Salter EM: Preoperative anthropometric dysmorphology in metopic synostosis. Am J Phys Anthropol 103:341-351, 1997

10. Loveday BP, de Chalain TB: Active counterpositioning or orthotic device to treat positional plagiocephaly? J Craniofac Surg 12:308-313, 2001

11. Mehta VA, Bettegowda C, Jallo GI, Ahn ES: The evolution of surgical management for craniosynostosis. Neurosurg Focus 29(6):E5, 2010

12. Mortenson PA, Steinbok P: Quantifying positional plagiocephaly: reliability and validity of anthropometric measurements. J Craniofac Surg 17:413-419, 2006

13. Schaaf H, Wilbrand JF, Boedeker RH, Howaldt HP: Accuracy of photographic assessment compared with standard anthropometric measurements in nonsynostotic cranial deformities. Cleft Palate Craniofac J 47:447-453, 2010

14. Vu HL, Panchal J, Parker EE, Levine NS, Francel P: The timing of physiologic closure of the metopic suture: a review of 159 patients using reconstructed 3D CT scans of the craniofacial region. J Craniofac Surg 12:527-532, 2001

15. Whitaker LA, Bartlett SP, Schut L, Bruce D: Craniosynostosis: an analysis of the timing, treatment, and complications in 164 consecutive patients. Plast Reconstr Surg 80:195-212, 1987

16. Wilbrand JF, Schmidtberg K, Bierther U, Streckbein P, PonsKuehnemann J, Christophis P, et al: Clinical classification of infant nonsynostotic cranial deformity. J Pediatr 161:11201125,2012 
17. Wilbrand JF, Wilbrand M, Pons-Kuehnemann J, Blecher JC, Christophis P, Howaldt HP, et al: Value and reliability of anthropometric measurements of cranial deformity in early childhood. J Craniomaxillofac Surg 39:24-29, 2011

\section{Disclosures}

The authors report no conflict of interest concerning the materials or methods used in this study or the findings specified in this paper.

\section{Author Contributions}

Conception and design: Ahn, Pindrik, Dorafshar. Acquisition of data: Pindrik, Molenda. Analysis and interpretation of data: Ahn, Pindrik, Molenda. Drafting the article: Pindrik, Uribe-Cardenas. Critically revising the article: all authors. Reviewed submitted version of manuscript: all authors. Approved the final version of the manuscript on behalf of all authors: Ahn. Statistical analysis: Pindrik. Administrative/technical/material support: Ahn. Study supervision: Ahn.

\section{Supplemental Information}

\section{Previous Presentations}

Portions of this work were presented in oral form at the 81st American Association of Neurological Surgeons Annual Scientific Meeting (Pediatric Neurosurgery Section), New Orleans, Louisiana, April 2013.

\section{Correspondence}

Edward S. Ahn, Division of Pediatric Neurosurgery, Johns Hopkins University School of Medicine, 600 North Wolfe St., Phipps 560A, Baltimore, MD 21287. email: eahn4@jhmi.edu. 